\title{
Article
}

\section{High-Risk Clone of Klebsiella pneumoniae Co-Harbouring Class $A$ and D Carbapenemases in Italy}

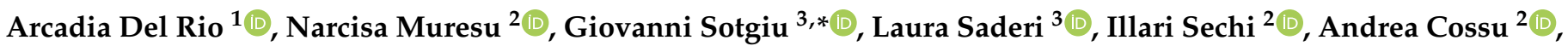

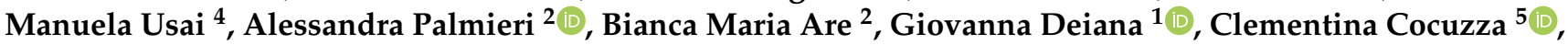 \\ Marianna Martinelli ${ }^{5}{ }^{-}$, Enrico Calaresu ${ }^{5}$ and Andrea Fausto Piana ${ }^{2} \mathbb{D}$
}

check for

updates

Citation: Del Rio, A.; Muresu, N.;

Sotgiu, G.; Saderi, L.; Sechi, I.; Cossu, A.; Usai, M.; Palmieri, A.; Are, B.M.; Deiana, G.; et al. High-Risk Clone of Klebsiella pneumoniae Co-Harbouring Class A and D Carbapenemases in Italy. Int. J. Environ. Res. Public Health 2022, 19, 2623. https://doi.org/ 10.3390/ijerph19052623

Academic Editor: Paul B. Tchounwou

Received: 24 January 2022

Accepted: 22 February 2022

Published: 24 February 2022

Publisher's Note: MDPI stays neutral with regard to jurisdictional claims in published maps and institutional affiliations.

Copyright: (C) 2022 by the authors. Licensee MDPI, Basel, Switzerland. This article is an open access article distributed under the terms and conditions of the Creative Commons Attribution (CC BY) license (https:// creativecommons.org/licenses/by/ $4.0 /)$.
1 Biomedical Science PhD School, Biomedical Science Department, University of Sassari, 07100 Sassari, Italy; delrio.arcadia2@gmail.com (A.D.R.); giovanna.deiana90@gmail.com (G.D.)

2 Hygiene Unit, Department of Medical, Surgical and Experimental Sciences, University of Sassari, 07100 Sassari, Italy; narcisamuresu@outlook.com (N.M.); illasechi@uniss.it (I.S.); andreacossu@uniss.it (A.C.); luca@uniss.it (A.P.); bianca.are@aousassari.it (B.M.A.); piana@uniss.it (A.F.P.)

3 Clinical Epidemiology and Medical Statistics Unit, Department of Medical, Surgical and Experimental Sciences, University of Sassari, 07100 Sassari, Italy; 1saderi@uniss.it

4 Department of Humanistic and Social Sciences, University of Sassari, 07100 Sassari, Italy; manuelausai@hotmail.com

5 Department of Medicine and Surgery, University of Milano-Bicocca, 20900 Monza, Italy; clementina.cocuzza@unimib.it (C.C.); marianna.martinelli@unimib.it (M.M.); e.calaresu1@gmail.com (E.C.)

* Correspondence: gsotgiu@uniss.it; Tel.: +39-079-229959

\begin{abstract}
Background: Carbapenem-resistant Klebsiella pneumoniae (CR-Kp) is endemic globally, causing severe infections in hospitalized patients. Surveillance programs help monitor and promptly identify the emergence of new clones. We reported the rapid spread of a novel clone of K. pneumoniae co-harbouring class A and D carbapenemases in colonized patients, and the potential risk factors involved in the development of infections. Methods: Rectal swabs were used for microbiological analyses and detection of the most common carbapenemase encoding genes by real-time PCR (i.e., blaKPC, blaOXA-48, blaNDM, blaVIM, and blaIMP). All strains co-harbouring KPC and OXA-48 genes were evaluated. For each patient, the following variables were collected: age, sex, length and ward of stay, device use, and outcome. Clonality of CR-Kp was assessed by preliminary pulsed field gel electrophoresis (PFGE), followed by multi-locus sequence typing (MLST) analyses. Results: A total of 127 isolates of K. pneumoniae co-harbouring KPC and OXA-48 were collected between September 2019 and December 2020. The median age (IQR) of patients was 70 (61-77). More than $40 \%$ of patients were admitted to intensive care unit (ICU). Around $25 \%$ of patients developed an invasive infection, the majority of which were respiratory tract infections $(17 / 31 ; 54.8 \%)$. ICU stay and invasive infection increased the risk of mortality (OR: 5.39, 95\% CI: 2.42-12.00; OR 6.12, 95\% CI: $2.55-14.69$, respectively; $p$-value $\leq 0.001)$. The antibiotic susceptibility test showed a resistance profile for almost all antibiotics considered. Monoclonal origin was confirmed by PFGE and MLST showing a similar restriction pattern and belonging to ST-512. Conclusions: We report the spread and the marked antibiotic resistance profiles of K. pneumoniae strains co-producing KPC and OXA-48. Further study could clarify the roles of clinical and microbiological variables in the development of invasive infection and increasing risk of mortality, in colonized patients.
\end{abstract}

Keywords: antimicrobial resistance; Carbapenemase genes; Klebsiella pneumoniae; nosocomial infections

\section{Introduction}

Hospital-acquired infections (HAIs) are associated with significant morbidity and mortality [1]. More than 4 million cases of HAIs occur annually in Europe, with 37,000 deaths [2], and $>60 \%$ of them are caused by multi-drug resistant (MDR) microorganisms, such as methicillin-resistant Staphylococcus aureus (MRSA), extended-spectrum beta-lactamase 
(ESBL)-producing bacteria, carbapenem-resistant Enterobacteriaceae (CRE), carbapenemresistant Acinetobacter baumannii, and MDR Pseudomonas aeruginosa [2,3].

The high MDR rate, especially in nosocomial settings, can reduce the availability of effective therapeutic options, increasing mortality and healthcare costs. Mortality associated with CRE ranges from 30\% to $70 \%$ and exceeds $50 \%$ in case of bloodstream infections. Carbapenems act as inhibitors in the synthesis of the bacterial cell wall, binding to penicillinbinding proteins. In the past, carbapenems were often recognized as "last-line agents" in the treatment of infections caused by Gram-negative and Gram-positive bacteria, based on their broad spectrum of activity. Among the acquired mechanisms of resistance, the inactivation of antimicrobial drugs following the production of carbapenemases is one of the most frequent mechanisms of resistance [4,5]. Carbapenem-resistant K. pneumoniae (CR-Kp) is endemic in USA, Brazil, Argentina, Colombia, China, Taiwan, and Europe. Class A carbapenemases target the most prevalent $\beta$-lactamases (Klebsiella pneumoniae carbapenemases, KPCs) found in CR-Kp isolates. Class B metallo $\beta$-lactamases are encoded by VIM (Verona integron-encoded metallo- $\beta$-lactamase), IMP (Imipenemase metallo- $\beta$ lactamase), and NDM (New-Delhi metallo- $\beta$-lactamase) genes. Class D genes include OXA-48 carbapenemases, which show high activity against penicillins, low activity against carbapenems, and weak activity against broad-spectrum cephalosporins [6].

CRE are frequently transmitted through healthcare personnel's hands or contaminated medical equipment or surfaces [7].

Intestinal colonization by CRE can be a risk factor for infections (respiratory tract, urinary tract, bloodstream, surgical wounds, skin, and soft tissue) in hospitalized patients, with an associated mortality rate ranging from $30 \%$ to $75 \%$ [5].

Identification of carriers and adoption of containment measures are key for the prevention of further colonization cases and clinical outbreaks through the implementation of surveillance programs [8].

After the first detection of KPC-producing K. pneumoniae in 2015 at the University Hospital of Sassari, Italy [9], a surveillance program was implemented: rectal swabs upon hospital admission and screening for close contacts of index cases were systematically carried out [10]. In the present study we describe the epidemiological characteristics of a novel clone of K. pneumoniae co-harbouring KPC and OXA-48 carbapenemases isolated from colonized patients, and the associations between demographic/clinical variables and invasive infection and death. The findings could help plan appropriate infection control measures and preventive interventions.

\section{Materials and Methods}

An observational retrospective study was carried out at the University of Sassari, Italy. We considered all strains of K. pneumoniae co-producing KPC and OXA-48, since first detection in September 2019 to December 2020, isolated during the activities of the screening program. Only the first positive rectal swabs, collected during the activities of the screening program, were selected for the present study.

Colonization status was defined as a detection of CRE from rectal swab or faeces without any evidence of active infection, whereas infection, refers to the detection of carbapenem-resistant K. pneumoniae in patients with infection-related symptoms, isolated from clinical specimens or a normally sterile site (i.e., blood or bronchoalveolar lavage, respectively).

The following variables were retrospectively collected: demographics (sex, age), comorbidities, history of community or healthcare-associated infections, ward of admission, length of hospital stay, use of devices, and final clinical outcome.

\subsection{Microbiological Analysis}

Rectal swabs were plated into Chromid Carba Smart Agar (bioMérieux, Italia) [11]; after overnight incubation, identification was performed using the Vitek-2 System (bioMerieux, Marcy l'Etoile, France) [12]. Carbapenemase encoding genes, blaKPC, blaNDM, blaVIM, 
blaOXA-48, and blaIMP were detected by real time-PCR using the commercial kit Allplex Entero-DR assay. Antimicrobial resistance profile and clonality of CR-Kp strains were assessed based on a representative set of isolates, selected by ward of admission and time of detection. Drug susceptibility test and minimum inhibitory concentration (MIC) values were assessed referring to breakpoints of the European Committee on Antimicrobial Susceptibility Testing (EUCAST) [13]. Clonality was preliminarily investigated by pulsed-field gel electrophoresis (PFGE) and interpreted according to Tenover criteria [14]. Bacteria were suspended in agarose disk before DNA extraction and purification. Bacterial genomes were cut by ApaI restriction enzyme, and single fragments were separated by agarose PFGE using a Clamped Homogeneous Electric Fields DRII System (BIORAD). Images of gels were captured by Image Master Program, and positions of electrophoretic bands and the phylogenetic dendrograms were obtained by GelCompar II (Applied Math) (Supplementary Figure S1) [9]. Isolates were clonally related if the Dice coefficient was $>80 \%$, whereas patterns with indistinguishable PFGE banding patterns (similarity coefficient $>97 \%$ ) belonged to the same subtype. Multi-locus sequence typing (MLST) analysis was performed to measure genetic relatedness and sequence variation between alleles, by amplifying and sequencing the internal fragments of seven K. pneumoniae housekeeping genes: gapA, infB, mdh, pgi, phoE, rpoB, and tonB (Supplementary information, S2: MLST analyses). Sequence analysis was carried out using the software Bioedit, and each single locus was compared with those included in the database of the Pasteur Institute to evaluate percentage similarity and compatibility [15].

\subsection{Statistical Analysis}

The following variables were collected on an electronic form: demographic (i.e., sex, age), clinical (i.e., hospital and length of stay, use of device, comorbidities, outcome), and microbiological (i.e., occurrence of invasive infection, antimicrobial susceptibility testing, MLST, molecular analyses for carbapenemase detection) variables. Absolute and relative (\%) frequencies, means and standard deviations (SD), or medians and interquartile ranges (IQR) were used to summarize qualitative and normally and non-normally distributed quantitative variables, respectively. Data were analysed using STATA Version 17 (StataCorp, College Station, TX, USA).

\section{Results}

A total of 127 isolates of Klebsiella pneumoniae co-harbouring KPC and OXA-48 were detected between September 2019 and December 2020 at the University Hospital of Sassari, Italy. Fifty-three (41.7\%) patients were female, and the median (IQR) age was $70(61-77)$ years (Table 1$)$.

Table 1. Demographic characteristics of patients involved in the study $(n=127)$.

\begin{tabular}{|c|c|c|}
\hline \multicolumn{3}{|c|}{ Variables } \\
\hline \multicolumn{2}{|c|}{ Female, $n(\%)$} & $53(41.7)$ \\
\hline \multicolumn{2}{|c|}{ Median (IQR) age, years } & $70(61-77)$ \\
\hline \multirow{5}{*}{ Ward of admission, $n(\%)$} & Surgical ward & $38(29.9)$ \\
\hline & $\mathrm{ICU}$ & $51(40.2)$ \\
\hline & Medicine ward & $31(24.4)$ \\
\hline & Infectious diseases & $3(2.4)$ \\
\hline & Long-term care & $4(3.2)$ \\
\hline \multicolumn{2}{|c|}{ Median (IQR) length of stay, days } & $46(29-68)$ \\
\hline \multicolumn{2}{|c|}{ Device use, $n(\%)$} & $103(81.1)$ \\
\hline \multicolumn{2}{|c|}{ Median (IQR) No. devices } & $2(1-3)$ \\
\hline
\end{tabular}


Table 1. Cont.

\begin{tabular}{|c|c|c|}
\hline \multicolumn{3}{|c|}{ Variables } \\
\hline \multirow{8}{*}{ Device, $n(\%)$} & CVC & $68(66.0)$ \\
\hline & Peripheral venous catheters & $4(3.9)$ \\
\hline & Urinary catheters & $7(6.8)$ \\
\hline & PEG & $9(8.7)$ \\
\hline & Arterial line & $37(35.9)$ \\
\hline & VAP & $51(49.5)$ \\
\hline & Tracheal tube & $31(30.1)$ \\
\hline & Other stomia & $15(14.5)$ \\
\hline \multicolumn{2}{|c|}{ Median (IQR) number of days between admission and colonization } & $26(13-43)$ \\
\hline \multicolumn{2}{|c|}{ Subjects with comorbidity, $n(\%)$} & $84(66.1)$ \\
\hline \multirow{2}{*}{ Invasive infections, $n(\%)$} & Bloodstream infections & $14 / 31(45.2)$ \\
\hline & Respiratory tract infections & $17 / 31(54.8)$ \\
\hline \multicolumn{2}{|c|}{$\begin{array}{l}\text { Median (IQR) number of days between diagnosis of colonization and } \\
\text { invasive infection }\end{array}$} & $10(0-22)$ \\
\hline \multicolumn{2}{|c|}{ Deaths, $n(\%)$} & $42(33.1)$ \\
\hline
\end{tabular}

Patients were frequently admitted to the ICU $(51 / 127 ; 40.2 \%)$, surgical wards $(38 / 127$; $29.9 \%)$, internal medicine wards $(31 / 127 ; 24.4 \%)$, long-term care wards $(4 / 127 ; 3.2 \%)$, and the infectious diseases ward $(3 / 127 ; 2.4 \%)$ (Figure 1$)$. Median (IQR) length of hospital stay was 46 (29-68) days.

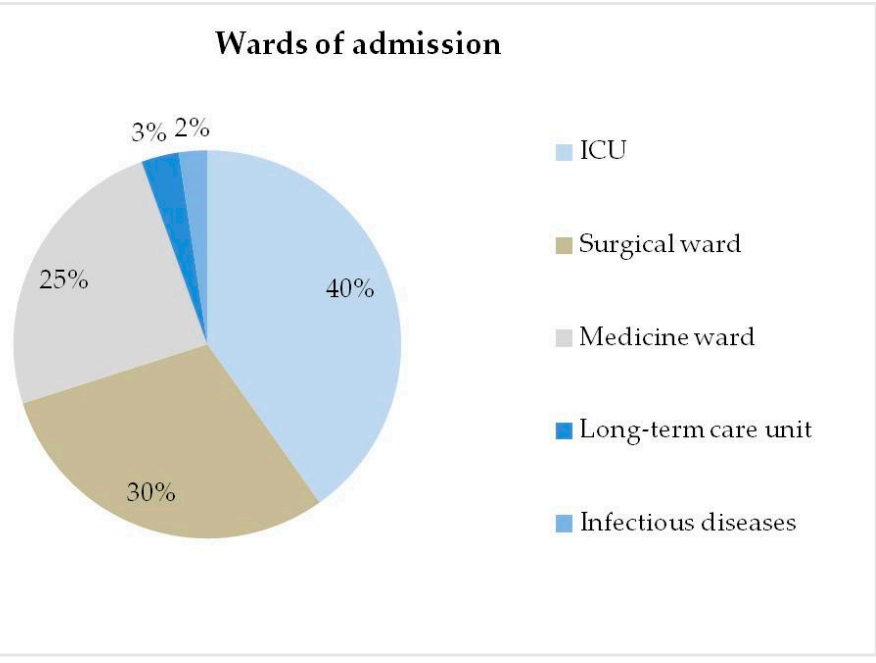

Figure 1. Representation of the distribution of wards of admission during the study period.

Over $80 \%(103 / 127 ; 81.1 \%)$ of patients had at least one clinical device: central venous catheter $(68 / 127,66.0 \%)$, mechanical ventilation $(51 / 127,49.5 \%)$, arterial line $(37 / 127$, $35.9 \%)$, and tracheal tube $(31 / 127,30.1 \%)$. The median (IQR) number of devices per patient was two (1-3).

The median (IQR) number of days between hospital admission and first detection of K. pneumoniae KPC-OXA-48 positive swab was 26 (13-43) days.

More than half the patients $(84 / 127,66.1 \%)$ had at least one comorbidity, including hypertension $(37,44.1 \%)$, cancer $(22,26.2 \%)$, diabetes $(21,25.0 \%)$, and cardiomyopathies $(20$, $23.8 \%$ ). Three quarters $(63 / 84 ; 75.0 \%$ ) of patients had two or more comorbidities (Table 2 ). 
Table 2. Prevalence of comorbidities.

\begin{tabular}{|c|c|c|c|}
\hline Comorbidity & $\begin{array}{l}\text { Total Cohort } n(\%) \\
\quad(84 ; 100 \%)\end{array}$ & $\begin{array}{c}\text { One Comorbidity } \\
(21 / 84 ; 25 \%)\end{array}$ & $\begin{array}{c}\geq 2 \text { Comorbidities } \\
(63 / 84 ; 75 \%)\end{array}$ \\
\hline Hypertension & $37(44.1)$ & $2(9.5)$ & $35(55.6)$ \\
\hline Cancer & $22(26.2)$ & $9(42.9)$ & $13(20.6)$ \\
\hline Diabetes & $21(25.0)$ & $2(9.5)$ & $19(30.2)$ \\
\hline Cardiomyopathy & $20(23.8)$ & $1(4.8)$ & $19(30.2)$ \\
\hline Atrial fibrillation & $14(16.7)$ & $0(0.0)$ & $14(22.2)$ \\
\hline Anaemia & $14(16.7)$ & $2(9.5)$ & $12(19.1)$ \\
\hline Obesity & $13(15.5)$ & $0(0.0)$ & $13(20.6)$ \\
\hline COPD & $12(14.3)$ & $0(0.0)$ & $12(19.1)$ \\
\hline Chronic renal failure & $12(14.3)$ & $1(4.8)$ & $11(17.5)$ \\
\hline Respiratory insufficiency & $10(11.9)$ & $1(4.8)$ & $9(14.3)$ \\
\hline $\begin{array}{l}\text { Benign prostatic } \\
\text { hyperplasia }\end{array}$ & $7(8.3)$ & $1(4.8)$ & $6(9.5)$ \\
\hline Asthma & $4(4.8)$ & $1(4.8)$ & $3(4.8)$ \\
\hline Auto-immune diseases & $4(4.8)$ & $0(0.0)$ & $4(6.4)$ \\
\hline Rheumatoid arthritis & $3(3.6)$ & $0(0.0)$ & $3(4.8)$ \\
\hline Multiple sclerosis & $3(3.6)$ & $1(4.8)$ & $2(3.2)$ \\
\hline Leukaemia/Myeloma & $2(2.4)$ & $0(0.0)$ & $2(3.2)$ \\
\hline
\end{tabular}

An invasive infection caused by KPC-OXA-48 K. pneumoniae occurred in 31/127 (24.4\%) patients; respiratory and bloodstream infections occurred in 17/31 (54.8\%) and $14 / 31(45.2 \%)$ patients, respectively. The median (IQR) number of days between a diagnosis of colonization and development of an invasive infection was 10 (0-22) days.

A total of $42(33.1 \%)$ deaths were reported. ICU stay was associated with an increased risk of mortality (OR: 5.39, 95\% CI: 2.42-12.00; $p$-value $\leq 0.001$ ) and an increased risk of invasive infection (OR 6.12, 95\% CI: 2.55-14.69; $p$-value < 0.001) (Table 3).

Table 3. Logistic regression analysis to assess the relationships between demographic, epidemiological and clinical variables and mortality.

\begin{tabular}{|c|c|c|c|}
\hline \multicolumn{2}{|c|}{ Variables } & OR $(95 \% \mathrm{CI})$ & $p$-Value \\
\hline \multicolumn{2}{|c|}{ Female, $n(\%)$} & $0.80(0.38-1.70)$ & 0.56 \\
\hline \multicolumn{2}{|c|}{ Median (IQR) age, years } & $1.03(1.00-1.06)$ & 0.07 \\
\hline \multirow{5}{*}{ Ward of admission, $n(\%)$} & Surgical ward & $0.16(0.05-0.48)$ & 0.001 \\
\hline & ICU & $5.39(2.42-12.00)$ & $<0.001$ \\
\hline & Medicine & $0.78(0.32-1.89)$ & 0.58 \\
\hline & Infectious diseases & / & / \\
\hline & Long-term care & $0.67(0.07-6.61)$ & 0.73 \\
\hline \multicolumn{2}{|c|}{ Median (IQR) length of stay, days } & $1.01(1.00-1.01)$ & 0.24 \\
\hline \multicolumn{2}{|c|}{ Device use } & $2.13(0.73-6.17)$ & 0.16 \\
\hline \multicolumn{2}{|c|}{ No. Devices } & $1.13(0.72-1.78)$ & 0.60 \\
\hline \multicolumn{2}{|c|}{$\begin{array}{l}\text { Median (IQR) number of days between admission } \\
\text { and positivity }\end{array}$} & $1.00(0.99-1.02)$ & 0.64 \\
\hline \multicolumn{2}{|c|}{ Subject with comorbidity, $n(\%)$} & $0.88(0.41-1.92)$ & 0.76 \\
\hline
\end{tabular}


Table 3. Cont.

\begin{tabular}{|c|c|c|c|}
\hline \multicolumn{2}{|c|}{ Variables } & OR (95\% CI) & $p$-Value \\
\hline \multirow{9}{*}{ Comorbidity, $n(\%)$} & Hypertension & $1.28(0.51-3.20)$ & 0.60 \\
\hline & Cardiomyopathy & $0.64(0.20-1.98)$ & 0.44 \\
\hline & Diabetes & $1.88(0.67-5.21)$ & 0.23 \\
\hline & COPD & $1.07(0.29-3.90)$ & 0.92 \\
\hline & Obesity & $0.93(0.26-3.33)$ & 0.91 \\
\hline & Asthma & l & l \\
\hline & Cancer & $0.98(0.35-2.78)$ & 0.97 \\
\hline & Respiratory insufficiency & / & / \\
\hline & Multiple sclerosis & / & / \\
\hline \multicolumn{2}{|c|}{$\begin{array}{l}\text { Median (IQR) number of days between diagnosis of } \\
\text { colonization and invasive infection }\end{array}$} & $0.99(0.98-1.01)$ & 0.35 \\
\hline \multicolumn{2}{|c|}{ Invasive infections, $n(\%)$} & $6.12(2.55-14.69)$ & $<0.001$ \\
\hline
\end{tabular}

Logistic regression analysis was carried out to assess the roles of demographic, clinical, and epidemiological factors in the occurrence of infection. It showed that diabetic patients (OR: 3.26, 95\% CI: 1.08-990; $p$-value: 0.04 ) and ICU stay (OR: 3.81, 95\% CI: 1.63-8.93; $p$-value: 0.002 ) were significantly associated with infection (Table 4 ).

Table 4. Logistic regression analysis to assess the relationships between demographic, epidemiological and clinical variables and development of invasive infections.

\begin{tabular}{|c|c|c|c|}
\hline \multicolumn{2}{|c|}{ Variables } & OR $(95 \% \mathrm{CI})$ & $p$-Value \\
\hline \multicolumn{2}{|c|}{ Female, $n(\%)$} & $0.48(0.20-1.16)$ & 0.10 \\
\hline \multicolumn{2}{|c|}{ Median (IQR) age, years } & $0.98(0.96-1.01)$ & 0.22 \\
\hline \multirow{5}{*}{ Ward of admission, $n(\%)$} & Surgical ward & $0.27(0.09-0.84)$ & 0.02 \\
\hline & ICU & $3.81(1.63-8.93)$ & 0.002 \\
\hline & Medicine & $0.88(0.34-2.29)$ & 0.79 \\
\hline & Infectious diseases & I & I \\
\hline & Long-term care & / & / \\
\hline \multicolumn{2}{|c|}{ Median (IQR) length of stay, days } & $1.01(1.00-1.02)$ & 0.05 \\
\hline \multicolumn{2}{|c|}{ Device use } & $4.31(0.95-19.51)$ & 0.06 \\
\hline \multicolumn{2}{|c|}{ No. Devices } & $1.41(0.89-2.31)$ & 0.18 \\
\hline \multicolumn{2}{|c|}{$\begin{array}{c}\text { Median (IQR) number of days between admission } \\
\text { and positivity }\end{array}$} & $1.00(0.99-1.01)$ & 0.93 \\
\hline \multicolumn{2}{|c|}{ Subject with comorbidity, $n(\%)$} & $0.63(0.27-1.45)$ & 0.28 \\
\hline \multirow{9}{*}{ Comorbidity, $n(\%)$} & Hypertension & $1.81(0.63-5.17)$ & 0.27 \\
\hline & Cardiomyopathy & $0.58(0.15-2.23)$ & 0.43 \\
\hline & Diabetes & $3.26(1.08-9.90)$ & 0.04 \\
\hline & COPD & $0.70(0.14-3.53)$ & 0.67 \\
\hline & Obesity & $1.12(0.27-4.59)$ & 0.88 \\
\hline & Asthma & / & / \\
\hline & Cancer & $0.76(0.22-2.62)$ & 0.67 \\
\hline & Respiratory insufficiency & / & / \\
\hline & Multiple sclerosis & / & / \\
\hline
\end{tabular}


The antibiotic susceptibility test, performed for 67 (52.8\%) isolates, showed a complicated drug resistance pattern to almost all classes of antibiotics, including carbapenems, cephalosporins, penicillins, and aminoglycosides (Table 5).

Table 5. Results of drug susceptibility testing of Klebsiella pneumoniae co-harbouring KPC and OXA-48.

\begin{tabular}{|c|c|c|}
\hline \multirow[t]{2}{*}{ Antibiotic } & \multicolumn{2}{|c|}{ Number of Isolates (\%): } \\
\hline & Intermediately Resistant & Resistant \\
\hline Amikacin & - & $69 / 69(100)$ \\
\hline Amoxicillin/Clavulanic acid & - & $67 / 67(100)$ \\
\hline Cefepime & - & $38 / 38(100)$ \\
\hline Cefotaxime & - & $69 / 69(100)$ \\
\hline Ceftazidime & - & $69 / 69(100)$ \\
\hline Ciprofloxacin & - & $69 / 69(100)$ \\
\hline Ertapenem & - & $48 / 48(100)$ \\
\hline Fosfomycin & - & $36 / 36(100)$ \\
\hline Gentamicin & - & $65 / 69(94)$ \\
\hline Imipenem & - & $25 / 25(100)$ \\
\hline Meropenem & $1 / 69(1)$ & $68 / 69(99)$ \\
\hline Piperacillin/Tazobactam & - & $67 / 67(100)$ \\
\hline Tigecycline & $4 / 32(12.5)$ & $28 / 32(88)$ \\
\hline Trimethoprim/Sulfamethoxazole & - & $67 / 67(100)$ \\
\hline Ceftazidime/Avibactam & - & $6 / 21(29)$ \\
\hline Ceftolozane/Tazobactam & - & $21 / 21(100)$ \\
\hline Tobramycin & - & $23 / 23(100)$ \\
\hline
\end{tabular}

A total of 44 representative samples of KPC and OXA-48 co-producing Klebsiella pneumoniae strains were analysed with PFGE. The PFGE analysis showed a similar restriction pattern; all strains were related to the same subtype (Dice coefficient $>80 \%$; similarity coefficient $>97 \%$ ). The MLST analysis showed a monoclonal origin of isolates, which belonged to the same sequence type, i.e., ST-512.

\section{Discussion}

The present study describes phenotypic and molecular characteristics of K. pneumoniae co-harbouring KPC and OXA-48, isolated at the university hospital of Sassari, Italy. A patient colonized by K. pneumoniae co-producing KPC and OXA-48 and transferred from a hospital located in northern Italy to the ICU of the university hospital of Sassari was the index case: the patient was immediately associated with a new case of colonization in a close contact and with rapid spread in the hospital wards. To the best of our knowledge, this is the first report of K. pneumoniae co-harbouring KPC and OXA-48 carbapenemases in Italy.

The incidence of CPE (carbapenemase producing Enterobacteriaceae) in Italy is high: almost one third of Klebsiella pneumoniae strains isolated from invasive infections are carbapenem-resistant, and the most common gene of resistance is KPC [16,17]. The KPC family includes more than 40 variants characterized by activity against cephalosporins, monobactams, and carbapenems [18]. Moreover, although the Italian prevalence is only $0.5 \%$ [17], class D carbapenemase OXA-48 is the most prevalent carbapenemase in Enterobacterales isolated in North Africa and in the Mediterranean area [19]. Class D carbapenemases showed high activity against aztreonam, extended spectrum cephalosporins, and carbapenems, especially when combined with other resistance mechanisms (i.e., re- 
duced permeability of membrane, and co-production of ESBL encoding genes or AmpC enzymes) [19].

Several reports show the worldwide emergence of strains with double or multiple carbapenemases, reducing the available treatment options, mostly in geographical areas where the endemicity of carbapenemase-producing Enterobacteriaceae is relevant [20]. Recently, Chen and Colleagues described the rapid spread of Klebsiella pneumoniae ST11 coexpressing class A and D carbapenemases in a teaching hospital in Taiwan [21]. The majority of carbapenemases genes can be transferred horizontally by mobile genetic elements, facilitating the evolutionary success of multiple-carbapenemase-producing strains, mainly in hospital settings where continuous antimicrobial resistance pressure occurs [22].

Antibiotic susceptibility tests performed in our setting showed a complicated drug resistance pattern. If compared with previous data on KPC-producing K. pneumoniae strains [9], the percentages of resistance to aminoglycosides and to tigecycline raised from $28 \%$ to $94.2 \%$ and from $50 \%$ to $87.5 \%$, respectively. This finding could potentially be explained by the co-expression of class $\mathrm{A}$ and $\mathrm{D}$ carbapenemases or by the presence of other additional genes localized in plasmids or mobile genetic elements [22]. Meletis and colleagues assumed that not all carbapenemases effectively hydrolyse the available betalactams; however, a combination of double or multiple carbapenemases causes an enhanced effect, favouring total resistance [20]. Moreover, resistance to ceftazidime/avibactam (CAZ/AVI) was observed in one third of the strains, raising concerns about the spread of emerging clones resistant to new therapeutic options. CAZ/AVI is active against class $\mathrm{A}, \mathrm{C}$, and D carbapenemases; and it is recommended for complicated urinary tract and intra-abdominal infections, and hospital-acquired and ventilator associated pneumonia caused by Gram-negative bacteria, including CR-Kp [23]. Haidar et al. [24] showed that mutations in KPC-3 variants could explain the higher MICs of CAZ/AVI and carbapenems. On this basis, the emergence of CAZ/AVI-resistant strains could be related to the presence of KPC mutants. However, further investigations could clarify the role of mutations behind the emergence of CAZ/AVI resistance.

We found an association between mortality and ICU stay, in agreement with the scientific literature focused on the roles played by some variables described in ICU wards (e.g., use of devices and prolonged antimicrobial therapies), as well-established risk factors for acquisition of CRE and higher mortality [25,26]. Moreover, higher risks of mortality and onset of infection in patients hospitalized in surgical units were associated with invasive and surgical procedures [27]. Although the mechanisms involved in the progression from colonization to infection are unclear, gastrointestinal carriage of K. pneumoniae is a wellestablished risk factor for invasive infections [28,29], as we observed in $>20 \%$ of colonized patients. The small samples size could have affected the identification of statistically significant associations between demographic variables (i.e., age and gender), several comorbidities, and outcomes. However, we found significant associations between diabetes and the onset of invasive infections. Several reports highlighted the role of underlying diseases in the progression from colonization to infection: they can weaken the immune response and increasing host susceptibility to infection [28]. Further studies are needed to explain how progression occurs and potential protective factors.

Unfortunately, missing data on previous or ongoing antimicrobial therapies did not allow us to evaluate the role of antibiotics in the onset of colonization or infections. However, interventions of antimicrobial stewardship could reduce the use of antimicrobial drugs, and then prevent the emergence of new antimicrobial resistant strains [30].

The mortality in our cohort was $33.1 \%$, increasing to $50 \%$ in the ICU, in agreement with previous reports which reported a mortality rate $>50 \%$ because of severe disease and poor therapeutic options [28].

A greater length of stay was associated with patients who developed invasive infections compared to colonized patients (46 VS. 71 days, respectively). Prolonged hospital stays are well-known risk factors for HAI, severe disease, death, and increased healthcare costs [25]. European guidelines strongly recommend screening swabs at hospital 
admission, and weekly surveillance for patients at high risk of carriage of MDR bacteria; moreover, screening of case-contacts is a key tool to contain the spread of MDR infections [31]. Early detection of carbapenem resistant K. pneumoniae, implementation of control measures, and prompt isolation of colonized patients may considerably reduce morbidity and mortality rates.

PFGE and MLST confirmed monoclonal circulation of ST-512, supporting national prevalence data $[9,32]$ and explaining the hospital spread of $K$. pneumoniae co-harbouring KPC and OXA-48 strains. Moreover, the rapid global transmission of ST-258, which is different when compared with ST-512 by only one mutation in gap locus, highlights the roles of genetic characteristics which can increase pathogenicity and transmissibility [33].

The limitations of the present study are mainly related to the absence of some important clinical data, and its retrospective and mono-centre epidemiological nature. In addition, the small sample size limited the power of the statistical analysis, including the identification of the potential roles played by risk factors. Advanced techniques, such as plasmid analysis and whole genome sequencing, could be useful for describing epidemiological dynamics, although these are not yet recommended in the available guidelines for screening programs [31].

The results of our screening program helped define the main epidemiological and clinical characteristics of circulating strains, although future multi-centre studies should investigate the roles of clinical and epidemiological factors in the prognosis of colonized patients. Of note, the new clone of K. pneumoniae co-harbouring class A and D carbapenemases detected in our setting highlight the risk of new multi-drug resistant strains.

\section{Conclusions}

In conclusion, surveillance programs, reinforcement of contact precautions, and education of staff can reduce the impact of antimicrobial resistance globally. Moreover, molecular characterization of antimicrobial resistance determinants, such as carbapenemase encoding genes, and adoption of alternative "non-antibiotic" strategies are essential to tracking the spread of high-risk strains, to promptly providing appropriate tailored therapeutic options, and to meeting "one-health" goals.

Supplementary Materials: The following are available online at https://www.mdpi.com/article/10 .3390/ijerph19052623/s1, Figure S1: Dendrogram and electrophoretic bands of Klebsiella pneumoniae isolates, co-producing KPC and OXA-48 carbapenemases, S2: MLST analyses.

Author Contributions: Conceptualization, A.D.R., N.M., G.S., C.C. and A.F.P.; methodology, A.D.R., N.M., G.S., M.U., A.C. and A.F.P.; software, L.S., I.S., M.M. and E.C.; validation, G.S., C.C. and A.F.P.; formal analysis, A.D.R., N.M., A.C., A.P., B.M.A., M.U., I.S., M.M. and E.C; investigation, A.D.R., N.M., G.S., A.P., B.M.A., G.D. and A.F.P.; resources, A.D.R., N.M., A.C., M.U., I.S., M.M. and E.C.; data curation, A.D.R., N.M., L.S., G.S. and A.F.P.; writing-original draft preparation, A.D.R., N.M., G.S. and A.F.P.; writing-review and editing, C.C., A.F.P. and M.M.; visualization, A.D.R., N.M., G.S., M.U., I.S., L.S., A.C., B.M.A., A.P., G.D., C.C., M.M., E.C. and A.F.P.; supervision, G.S., C.C., B.M.A., A.P. and A.F.P. All authors have read and agreed to the published version of the manuscript.

Funding: This research received no external funding.

Institutional Review Board Statement: Ethical review and approval were waived for this study, being that Italian law does not mandate ethical approval for an observational retrospective study.

Informed Consent Statement: Patient consent was waived due to national decree published in studies (G.U No. 76 31/Mar/2008).

Data Availability Statement: The data is available in case it is requested for motivated reasons.

Acknowledgments: The Authors thank all the stakeholders directly and indirectly involved in this research.

Conflicts of Interest: The authors declare no conflict of interest. 


\section{References}

1. Cornejo-Juárez, P.; Vilar-Compte, D.; Pérez-Jiménez, C.; Namendys-Silva, S.; Sandoval-Hernández, S.; Volkow-Fernández, P. The impact of hospital-acquired infections with multidrug-resistant bacteria in an oncology intensive care unit. Int. J. Infect. Dis. 2015, 31, 31-34. [CrossRef] [PubMed]

2. Cassini, A.; Högberg, L.D.; Plachouras, D.; Quattrocchi, A.; Hoxha, A.; Simonsen, G.S.; Colomb-Cotinat, M.; Kretzschmar, M.E.; Devleesschauwer, B.; Cecchini, M.; et al. Attributable deaths and disability-adjusted life-years caused by infections with antibiotic-resistant bacteria in the EU and the European Economic Area in 2015: A population-level modelling analysis. Lancet Infect. Dis. 2019, 19, 56-66. [CrossRef]

3. Wang, M.; Wei, H.; Zhao, Y.; Shang, L.; Di, L.; Lyu, C.; Liu, J. Analysis of multidrug-resistant bacteria in 3223 patients with hospital-acquired infections (HAI) from a tertiary general hospital in China. Bosn. J. Basic Med. Sci. 2019, 19, 86-93. [CrossRef] [PubMed]

4. El-Gamal, M.I.; Brahim, I.; Hisham, N.; Aladdin, R.; Mohammed, H.; Bahaaeldin, A. Recent updates of carbapenem antibiotics. Eur. J. Med. Chem. 2017, 131, 185-195. [CrossRef]

5. European Centre for Disease Prevention and Control. Carbapenem-Resistant Enterobacteriaceae, Second Update-26 September 2019; ECDC: Stockholm, Sweden, 2019. Available online: https://www.ecdc.europa.eu/en/publications-data/carbapenem-resistantenterobacteriaceae-second-update (accessed on 6 July 2021).

6. Bush, K.; Jacoby, G.A. Updated Functional Classification of $\beta$-Lactamases. Antimicrob. Agents Chemother. 2010, 54, 969-976. [CrossRef]

7. Jarvis, W.R.; Munn, V.P.; Highsmith, A.K.; Culver, D.H.; Hughes, J.M. The Epidemiology of Nosocomial Infections Caused by Klebsiella pneumoniae. Infect. Control 1985, 6, 68-74. [CrossRef]

8. Ambretti, S.; Bassetti, M.; Clerici, P.; Petrosillo, N.; Tumietto, F.; Viale, P.; Rossolini, G.M. Screening for carriage of carbapenemresistant Enterobacteriaceae in settings of high endemicity: A position paper from an Italian working group on CRE infections. Antimicrob. Resist. Infect. Control 2019, 8, 136. [CrossRef]

9. Sotgiu, G.; Are, B.; Pesapane, L.; Palmieri, A.; Muresu, N.; Cossu, A.; Dettori, M.; Azara, A.; Mura, I.; Cocuzza, C.; et al. Nosocomial transmission of carbapenem-resistant Klebsiella pneumoniae in an Italian university hospital: A molecular epidemiological study. J. Hosp. Infect. 2018, 99, 413-418. [CrossRef]

10. Protocollo Operativo per la Sorveglianza e Gestione dei Pazienti con Colonizzazione/Infezione da Enterobacteriaceae CPE/CRE, 27 September 2019. AOU Sassari. Available online: https:/ / www.aousassari.it/documenti/11_393_20190328122930.pdf (accessed on 24 November 2021).

11. CHROMID®CARBA SMART Selective Chromogenic Media for the Screening of Carbapenemase-Producing Enterobacteriaceae (CPE). Available online: http://www.biomerieux-culturemedia.com/product/99-chromid-carba-smart (accessed on 12 August 2021).

12. VITEK®2 Microbial ID/AST Testing System. Available online: https://www.biomerieux-diagnostics.com/vitekr-2-0 (accessed on 12 August 2021).

13. EUCAST Guidelines for Detection of Resistance Mechanisms and Specific Resistances of Clinical and/or Epidemiological Importance. EUCAST. 2017. Available online: https:/ / www.eucast.org/resistance_mechanisms/ (accessed on 6 July 2021).

14. Tenover, F.C. Interpreting Chromosomal DNA Restriction Patterns Produced by Pulsed-Field Gel Electrophoresis: Criteria for Bacterial Strain Typing. J. Clin. Microbiol. 1995, 33, 2233-2239. [CrossRef]

15. Primers Used for Amplification and Sequencing (MLST). Available online: Bigsdb.web.pasteur.fr/klebsiella/klebsiella.html (accessed on 6 July 2021).

16. Data from the ECDC Surveillance Atlas-Antimicrobial Resistance. 2019. Available online: https://www.ecdc.europa.eu/en/ antimicrobial-resistance/surveillance-and-disease-data/data-ecdc (accessed on 12 August 2021).

17. Grundmann, H.; Glasner, C.; Albiger, B.; Aanensen, D.M.; Tomlinson, C.T.; Andrasević, A.T.; Cantón, R.; Carmeli, Y.; Friedrich, A.W.; Giske, C.G.; et al. Occurrence of carbapenemase-producing Klebsiella pneumoniae and Escherichia coli in the European survey of carbapenemase-producing Enterobacteriaceae (EuSCAPE): A prospective, multinational study. Lancet Infect. Dis. 2017, 17, 153-163. [CrossRef]

18. Naas, T.; Dortet, L.; Iorga, B.I. Structural and Functional Aspects of Class A Carbapenemases. Curr. Drug Targets 2016, 17, 1006-1028. [CrossRef] [PubMed]

19. Jean, S.-S.; Lee, W.-S.; Lam, C.; Hsu, C.-W.; Chen, R.-J.; Hsueh, P.-R. Carbapenemase-producing Gram-negative bacteria: Current epidemics, antimicrobial susceptibility and treatment options. Future Microbiol. 2015, 10, 407-425. [CrossRef] [PubMed]

20. Meletis, G.; Chatzidimitriou, D.; Malisiovas, N. Double- and multi-carbapenemase-producers: The excessively armored bacilli of the current decade. Eur. J. Clin. Microbiol. 2015, 34, 1487-1493. [CrossRef]

21. Chen, C.-M.; Guo, M.-K.; Ke, S.-C.; Lin, Y.-P.; Li, C.-R.; Vy Nguyen, H.T.; Wu, L.-T. Emergence and nosocomial spread of ST11 carbapenem-resistant Klebsiella pneumoniae co-producing OXA-48 and KPC-2 in a regional hospital in Taiwan. J. Med. Microbiol. 2018, 67, 957-964. [CrossRef] [PubMed]

22. van Loon, K.; Voor in 't holt, A.F.; Vos, M.C. A Systematic Review and Meta-analyses of the Clinical Epidemiology of CarbapenemResistant Enterobacteriaceae. Antimicrob. Agents Chemother. 2018, 62, e01730-17. [CrossRef] [PubMed]

23. Van Duin, D.; Bonomo, R.A. Ceftazidime/Avibactam and Ceftolozane/Tazobactam: Second-generation $\beta$-Lactam/ $\beta$-Lactamase Inhibitor Combinations. Clin. Infect. Dis. 2016, 63, 234-241. [CrossRef] [PubMed] 
24. Haidar, G.; Clancy, C.J.; Shields, R.K.; Hao, B.; Cheng, S.; Nguyen, M.H. Mutations in bla KPC-3 That Confer CeftazidimeAvibactam Resistance Encode Novel KPC-3 Variants That Function as Extended-Spectrum $\beta$-Lactamases. Antimicrob. Agents Chemother. 2017, 61, e02534-16. [CrossRef]

25. Papadimitriou-Olivgeris, M.; Marangos, M.; Fligou, F.; Christofidou, M.; Bartzavali, C.; Anastassiou, E.D.; Filos, K.S. Risk factors for KPC-producing Klebsiella pneumoniae enteric colonization upon ICU admission. J. Antimicrob. Chemother. 2012, 67, 2976-2981. [CrossRef]

26. Lusignani, L.S.; Presterl, E.; Zatorska, B.; Nest, M.V.D.; Diab-Elschahawi, M. Infection control and risk factors for acquisition of carbapenemase-producing enterobacteriaceae. A 5 year (2011-2016) case-control study. Antimicrob. Resist. Infect. Control 2020, 9, 18. [CrossRef]

27. McConville, T.H.; Sullivan, S.B.; Gomez-Simmonds, A.; Whittier, S.; Uhlemann, A.-C. Carbapenem-resistant Enterobacteriaceae colonization (CRE) and subsequent risk of infection and 90-day mortality in critically ill patients, an observational study. PLoS ONE 2017, 12, e0186195. [CrossRef]

28. Martin, R.M.; Cao, J.; Brisse, S.; Passet, V.; Wu, W.; Zhao, L.; Malani, P.N.; Rao, K.; Bachman, M.A. Molecular Epidemiology of Colonizing and Infecting Isolates of Klebsiella pneumoniae. mSphere 2016, 1, e00261-16. [CrossRef] [PubMed]

29. Gorrie, C.; Mirčeta, M.; Wick, R.R.; Edwards, D.J.; Thomson, N.; Strugnell, R.; Pratt, N.F.; Garlick, J.S.; Watson, K.M.; Pilcher, D.; et al. Gastrointestinal Carriage Is a Major Reservoir of Klebsiella pneumoniae Infection in Intensive Care Patients. Clin. Infect. Dis. 2017, 65, 208-215. [CrossRef] [PubMed]

30. Dickstein, Y.; Temkin, E.; Ben-David, D.; Carmeli, Y.; Schwaber, M.J. Antimicrobial use trends, Israel, 2012 to 2017. Eurosurveillance 2019, 24, 1900022. [CrossRef] [PubMed]

31. Tacconelli, E.; Cataldo, M.A.; Dancer, S.J.; De Angelis, G.; Falcone, M.; Frank, U.; Kahlmeter, G.; Pan, A.; Petrosillo, N.; Rodríguez-Baño, J.; et al. ESCMID guidelines for the management of the infection control measures to reduce transmission of multidrug-resistant Gram-negative bacteria in hospitalized patients. Clin. Microbiol. Infect. 2014, 20, 1-55. [CrossRef]

32. Conte, V.; Monaco, M.; Giani, T.; D’Ancona, F.; Moro, M.L.; Arena, F.; D’Andrea, M.M.; Rossolini, G.M.; Pantosti, A. Molecular epidemiology of KPC-producing Klebsiella pneumoniae from invasive infections in Italy: Increasing diversity with predominance of the ST512 clade II sublineage. J. Antimicrob. Chemother. 2016, 71, 3386-3391. [CrossRef]

33. Bellich, B.; Lagatolla, C.; Rizzo, R.; D'Andrea, M.M.; Rossolini, G.M.; Cescutti, P. Determination of the capsular polysaccharide structure of the Klebsiella pneumoniae ST512 representative strain KPB-1 and assignments of the glycosyltransferases functions. Int. J. Biol. Macromol. 2020, 155, 315-323. [CrossRef] 